\title{
Filigrane
}

Écoutes psychothérapiques

\section{Se hâter avec lenteur. Le défi des jeunes majeurs pris en charge par l'Aide sociale à l'enfance}

\section{Nicolas Peraldi}

Volume 22, numéro 1, printemps 2013

Psychanalyse et temporalités

URI : https://id.erudit.org/iderudit/1017343ar

DOI : https://doi.org/10.7202/1017343ar

Aller au sommaire du numéro

Éditeur(s)

Revue Santé mentale au Québec

ISSN

1192-1412 (imprimé)

1911-4656 (numérique)

Découvrir la revue

Citer cet article

Peraldi, N. (2013). Se hâter avec lenteur. Le défi des jeunes majeurs pris en charge par l'Aide sociale à l'enfance. Filigrane, 22(1), 11-25.

https://doi.org/10.7202/1017343ar
Résumé de l'article

Voyager entre Charybde et Scylla. L'expression homérique est connue. Entre les écueils souvent acérés des demandes qui leur sont adressées et la tempête de la subjectivation, les jeunes pris en charge par l'Aide sociale à l'enfance doivent se mesurer à deux temps, l'un social, l'autre psychique. L'un et l'autre ne suivant pas le même rythme. C'est dans ces mouvements que l'auteur essaie de penser avec ces jeunes sur leurs errements, et ce en fonction des décisions qui interviennent dans la direction de la cure. 


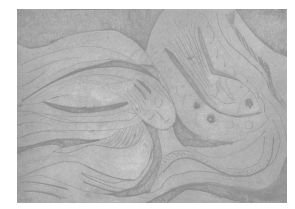

\title{
Se hâter avec lenteur. Le défi des jeunes majeurs pris en charge par l'Aide sociale à I'enfance $^{1}$
}

\section{Nicolas Peraldi}

\begin{abstract}
Voyager entre Charybde et Scylla. L'expression homérique est connue. Entre les écueils souvent acérés des demandes qui leur sont adressées et la tempête de la subjectivation, les jeunes pris en charge par I'Aide sociale à l'enfance doivent se mesurer à deux temps, I'un social, I'autre psychique. L'un et l'autre ne suivant pas le même rythme. C'est dans ces mouvements que l'auteur essaie de penser avec ces jeunes sur leurs errements, et ce en fonction des décisions qui interviennent dans la direction de la cure.
\end{abstract}

ierkegaard écrivait cette assertion, psychanalytique avant l'heure : «Parce que je me retourne vers le passé, je vois l'avenir. »

La conjonction «parce que» sert habituellement à introduire une cause, voire un mouvement, ici une direction, que l'on pourrait penser être celle de la cure, une gageure ou un engagement pour un sujet dans son histoire qui a pour effet de lui accorder une autre place. Analyse vient du grec analysis, qui signifie « dénouer». La vie des jeunes pris en charge par l'Aide sociale à l'enfance $^{2}$ est un véritable sac de nœuds. Ils en portent le poids, souvent sans le savoir.

Le travail d'un analyste avec un adolescent n'est pas en soi une mince affaire. Il ne veut rien en savoir de son histoire. L'adolescent, pas l'analyste... Il aimerait bien ne pas avoir à la ressasser une nouvelle fois. Et pourtant ce passé, si présent, l'encombre et l'envahit dans tous les espaces: vie familiale, vie sentimentale, vie scolaire ou sociale, professionnelle parfois. La liste n'étant bien sûr pas exhaustive.

Les temps se mêlent et se répètent. Le matin ressemble à la veille et donne une idée du lendemain. Rien de surprenant que nombre de ces jeunes à l'abandon ${ }^{3}$ procrastinent ${ }^{4}$ sans modération. Une des difficultés réside dans 
l'équilibre à créer entre ce temps en boucle et la dimension subjective qui s'y déploie, entre le temps social et le temps psychique. L'un et l'autre sont imbriqués, sans que le sujet ne le sache vraiment, mais ils ne vont pas nécessairement dans la même direction, d'où une tension sous forme de symptôme.

Si Chronos donne le rythme, il faut que Kairos vienne le scander pour lui donner une nouvelle impulsion. Chronos et Kairos, deux figures du temps dans la mythologie grecque, une double lecture de ce qui peut se jouer dans une cure, notamment avec des jeunes pris en charge par l'ASE.

\section{Chronos}

«Hier était le jour précédant aujourd'hui et demain sera le jour suivant parce que je suis aujourd'hui » pourrait dire Chronos. Il est le point mouvant sur la flèche du temps.

Je travaille avec des adolescents et des jeunes majeurs qui me sont adressés par des associations qui dépendent de l'ASE. Si une prise en charge peut commencer très tôt, certains enfants pouvant être placés réellement ${ }^{5}$ dès leur naissance, elle ne pourra jamais excéder l'âge de 21 ans ${ }^{6}$. C'est une date butoir qui, si elle a une histoire ${ }^{7}$, vient faire point d'arrêt pour toute prise en charge. Il va sans dire qu'une prise en charge peut s'arrêter bien évidemment avant cette date limite, lorsqu'elle n'est pas vécue comme "date de péremption ", pour différentes raisons, aussi objectives que subjectives. Entretemps, la prise en charge est scandée par le renouvellement de contrat tant avec l'association mandatée qu'avec l'administration qu'est l'ASE. Ces temps peuvent être ceux d'une mise au point, d'une évaluation de la pertinence de la prise en charge ou parfois d'un certain exercice du pouvoir... Quoi qu'il en soit, mon but n'étant pas dans cet article de remettre en question certaines pratiques, toute prise en charge s'inscrit dans un temps social qui se lit sur la flèche du temps qu'est Chronos. C'est une donnée incontournable, comme le temps lui-même, qui a son fondement et son utilité et avec laquelle je me dois de travailler en cela qu'elle est une des conditions de toute prise en charge, ou du moins qu'elle lui donne certains rythmes. Les temps, celui qui limite la prise en charge et ceux qui la scandent, infléchissent considérablement le cours du travail thérapeutique.

Dans le cadre de leur prise en charge, il est proposé à ces jeunes de rencontrer un psychanalyste en libéral à raison d'une à deux séances par semaine. Cette dynamique a pour fonction essentielle de leur permettre de mettre des mots sur ce qu'ils vivent, dans un cadre autre que celui institutionnel de la prise en charge; cadre soutenu par un relatif secret professionnel. Relatif en 
cela que je suis amené à travailler, ne serait-ce que ponctuellement, avec les équipes éducatives et leurs Directions, à écrire des comptes-rendus adressés à l'ASE ou des notes de situation. Et les jeunes le savent. Si cela ne leur a pas été dit, je le fais afin qu'ils sachent que je suis engagé ${ }^{8}$ dans la prise en charge. Mon travail se situe donc dans un espace intermédiaire entre la clinique libérale classique, et les logiques sociales et institutionnelles inhérentes à toute prise en charge. Si les histoires de ces jeunes sont complexes, leurs parcours institutionnels le sont tout autant et les rapports qu'ils entretiennent avec les différents professionnels sont marqués de ces complexités.

Souvent, les jeunes pris en charge ont un discours rodé. Leur parcours a déjà été balisé par différentes rencontres au cours desquelles ils ont pu, à loisir, parler de ce qui leur est arrivé, ou du moins de ce qu'ils veulent en dire, pour qu'ils se retrouvent pris en charge. Le redoublement de ce terme - pris en charge -, au début et à la fin de la narration, peut clore toute élaboration. C'est souvent le point de départ et celui d'arrêt.

Ils ont déjà rencontré des assistantes sociales, des éducateurs, des «psy» (avec toute la confusion que ce terme contient). Ils ont déjà dit et redit et ils savent parfois ce qu'il faut dire; parfois ou parfaitement. Ils nous versent la soupe, sans vouloir en manger. Mais ont-ils le choix de faire autrement? Être pris en charge est une nécessité impérieuse pour beaucoup, si ce n'est pour tous. Leur vie est à ce point chaotique qu'ils ne peuvent envisager une autre sortie, une autre résolution. Et un temps, ne serait-ce qu'un temps, ils sont prêts à accepter toutes les conditions du contrat, dont aller voir un "psy", même s'il nous faut ne pas être dupe pour autant. Il me semble éthique de ne pas trop en vouloir à ces jeunes, de les accompagner dans leurs errances et leurs errements afin qu'ils puissent au mieux trouver par eux-mêmes une résolution adéquate à leur problématique. "Ne pas trop leur en vouloir », c'est à la fois ne pas trop leur demander et ne pas trop leur reprocher de ne pas être tels que l'on voudrait qu'ils soient. Cela permet d'éviter cet écueil de la déception qui peut survenir dans une prise en charge. C'est pour cela qu'il ne faut pas être dupe du discours préétabli des jeunes qui font une demande de prise en charge. La plupart du temps, ils ne répondent pas à cette demande. Elle n'est qu'un leurre, une accroche. Le projet quant à lui, aussi nécessaire soit-il pour penser, n'est qu'un lieu commun, au sens figuré comme au sens propre.

Le travail avec un analyste ouvre parfois sur d'autres espaces qui permettent d'interroger les impasses subjectives et de faire émerger le désir du jeune. Un désir qui peut aller à l'encontre de ce qu'il avait préalablement avancé, de ce que l'on avait toujours attendu de lui ou de ce qu'il croyait, dupe 
de lui-même, devoir faire. Encore faut-il que ce désir se dise plutôt que de se faire entendre, parfois à corps et à cris ${ }^{9}$.

Mais parler c'est prendre des risques. Celui de décevoir, d'être rejeté, de se sentir isolé d'avoir voulu s'affirmer. Celui du malentendu, voire de l'incompréhension. Mais surtout, c'est celui de réveiller certaines blessures que l'on pensait cicatrisées ou du moins, suffisamment bien refoulées. À tel point que l'on mesure qu'elles ont toujours été là, ces blessures, sous des formes déguisées. C'est l'enjeu de la reconstruction discursive, de la façon dont un sujet va pouvoir entendre, dans son discours, ce qui fait impasse et semblant, afin de s'approprier son histoire pour sortir des ornières de la logique du préjudice inscrit en filigrane dans le terme d'usager ${ }^{10}$. L'usager est dépossédé de son histoire puisque celle-ci conditionne sa prise en charge. Si on le dit acteur, ce n'est que dans le cadre restreint de cette prise en charge, comme s'il ne l'avait pas été préalablement. L'écoute psychanalytique produit un contrepoint à cette logique sociale. Elle ramène, par son travail d'historicité, le symptôme au cour du sujet, en sa causalité psychique inconsciente. La question n'est donc pas d'établir l'Histoire du sujet, comme l'on ferait une chronologie des faits - même si ce travail d'investigation pourrait parfois s'avérer utile - mais de lui permettre de (se) raconter son histoire, de faire un travail d'historisation, travail qui accorde un savoir singulier sur le symptôme. Les impasses subjectives sont liées à l'histoire du jeune, même s'il n'en sait rien. Comme l'écrivait F. Dolto (1981): «au jeu du désir, les dés sont pipés et les cartes truquées. » Avant même sa naissance, un enfant est préalablement engagé, sans rien en savoir, dans les rets de désirs qui le concernent et avec lesquels il va devoir composer.

«Wo es war, soll Ich werden.» (Freud, 1932/1984, 110) La formule est connue. «Là où C'était, Je dois advenir » pourrait-on traduire. Le Je est, en un sens, cette histoire par laquelle le sujet qui advient se donne et nous donne une nouvelle version, sa version, qui se substitue à un temps écoulé et, comme tel, définitivement perdu.

Un «temps parlé» garantit la mémorisation d'un temps passé. C’est un présupposé pour l'existence d'un Je. Cette reconstruction du vécu est une condition nécessaire pour la mise en place d'un investissement du temps futur. Cette autobiographie peut transformer un temps psychique en temps humain, subjectif, qui fait sens et qui peut être investi. Toute la difficulté est d'harmoniser ce temps psychique au temps social de la prise en charge, ou plus exactement de faire rythmer ce temps psychique dans le temps social. C'est là le défi dans lequel les jeunes et les professionnels sont engagés. C'est là que Chronos et Kairos se rencontrent. 


\section{Kairos}

Kairos est une autre figure du temps. Il est représenté comme un jeune homme avec une natte qu'il faut saisir si possible au moment où il passe. Kairos est le moment opportun, l'occasion à saisir et comme tel, il est fugace.

«Le temps parlé» engage le discours parental auquel l'enfant s'était plus ou moins sagement assigné. Au moment où le sujet entre dans l'adolescence, au moment où il refuse de se considérer comme un enfant, il va commencer à modifier son passé. Dans son récit, il rendra son passé responsable de ce qu'il est et de ce qu'il a, comme de ce qu'il n'est pas et de ce qu'il n'a pas. Il devra en passer par ce défilé pour essayer de se construire. Mais l'essentiel reste néanmoins à faire. Il faut que de cette affirmation, parfois toute en négation, se créent les conditions nécessaires d'une rencontre qui permette l'élaboration d'un autre discours. Un discours qui ne soit plus celui de la plainte ou du préjudice. Un discours qui laisse émerger une parole qui puisse être entendue sans que le lien ne soit détruit. Et ce discours passe par une reconstruction dans laquelle le sujet parle de lui, pour lui, avec lui. Pour enfin, peutêtre, oublier?

Le travail analytique servirait-il donc à oublier? Curieuse question lorsque l'on sait tout le travail de remémoration qui est à l'ouvre dans ce processus. Mais d'une certaine façon, l'analyse servirait à oublier en payant d'abord le prix psychique pour se remémorer. Dès lors, tout vient à sa place. Cela permet de constater que la réminiscence est ce fragment de mémoire qui vagabonde entre l'Inconscient et la Conscience. Le souvenir est le premier pas vers la tentative d'oubli et en même temps, son principal obstacle.

Pour la psychanalyse, la mémoire est du côté de l'Inconscient: «Dans l'inconscient, rien ne peut être mené à sa fin, rien n'est passé ni oublié.» (Freud, 1900/2003, 633)

De toutes les mémoires, l'Inconscient est la plus vivante. L'humiliation subie dans l'enfance, pour peu qu'elle soit en contact avec du refoulé, peut agir des décennies plus tard comme si elle était de fraîche date. Quant au souvenir, il est du côté de l'écran. Il fixe des impressions comme une photographie fixe un instant. Il n'est pas rare de rencontrer ces patients dont toute l'enfance est un souvenir, un souvenir qui les prive pourtant de leur enfance.

Ce n'est que lorsque nous nous sommes rappelés assez souvent et assez clairement une expérience qu'elle peut échapper à notre mémoire. (Reik, 1976, 155) 
Le non remémoré est psychiquement immortel. Dans une certaine mesure, la reconstruction discursive induit des renoncements, crée les conditions nécessaires pour que des deuils de certains objets archaïques puissent se faire, pour que les évènements non remémorés puissent s'inscrire sur le fil du temps. On pourrait dire que le passé ne peut sombrer dans l'oubli que lorsqu’il est redevenu présent. Ce passé qui ne sombre qu'en étant présent, c'est ce que Freud a nommé le transfert.

Parler du transfert aujourd'hui tient de la résistance au discours dominant. Si je devais définir un des pans du transfert, et pas le moindre me semble-t-il, je dirais que c'est une double erreur. Erreur sur le temps de l'événement raconté et erreur sur la personne. Le passé se conjugue inconsciemment au présent, et les figures se superposent les unes aux autres sans que le sujet ne s'en aperçoive vraiment. Le jeune répète, depuis longtemps déjà, des éléments de son passé sans s'en rendre compte. Il est rejeté des différents placements, il échoue systématiquement dans ses études, ses relations sentimentales virent au désastre... Tout le travail tient, au moment opportun, à lui faire entendre ce qui se répète afin qu'il s'en rende compte et modifie son rapport à soi, aux autres et au monde. Ce moment opportun, ce temps d'interprétation, pour qu'il soit efficace et non intrusif, c'est-à-dire pour que le sujet puisse l'entendre, soit prêt à l'entendre, ce moment donc prend son temps avant d'apparaître. Et tel Kairos, il faut le saisir au moment où il passe.

«Le lion ne bondit qu'une fois.» (Freud, 1937/1985, 234) L'interprétation, à laquelle Freud se réfère dans cette métaphore, consiste donc, entre autre, à libérer le sujet de l'emprise de certains énoncés qui l'ont aliéné à une image qu'il avait de lui-même, ou plus exactement à faire cesser l'aliénation dans laquelle il se maintenait ${ }^{11}$.

La difficulté est que le sujet ne souhaite pas nécessairement changer. Il peut trouver une certaine sécurité, parfois même une assurance, dans ses symptômes, lesquels s'inscrivent toujours dans une histoire singulière. S'il est difficile de parler de guérison en psychanalyse, ce qui permet d'ailleurs de gonfler l'ire de ses détracteurs, l'on ne guérit pas d'être soi, on apprend à l'être au mieux avec ses fondements radicaux. La psychanalyse permet d'ajuster ses désirs en fonction d'une modification de la perception de la réalité. Elle atténue les conflits intrapsychiques afin de permettre aux jeunes d'être plus ou moins en adéquation avec le monde qui les entoure et qu'ils ne comprennent pas forcément. La psychanalyse fait sens, dans toute la polysémie de ce terme. Elle permet de porter le regard ailleurs que là où la crise surgit, de ne pas se laisser happer par le symptôme d'un jeune pris en charge. Le fait de ne pas 
porter toute son attention à ce symptôme, de ne pas se focaliser sur cette mise en scène permet de l'interroger dans la singularité d'une histoire. Voire de restituer l'histoire du symptôme, puisque celui ci ne vient pas de nulle part. Il a une histoire et une fonction.

"L'analyse ne guérit pas nécessairement d'un symptôme, elle guérit de la nécessité du symptôme. » (Zygouris, 2006, 17) Elle produit donc un changement psychique, et notamment modifie le rapport que l'on entretient avec le monde, avec les autres et avec soi. Le jeune pris en charge sort avec une autre histoire que celle dont il avait fait le récit inaugural. Une histoire autre et paradoxalement plus que jamais la sienne. La narration d'une histoire peut rattraper et modifier ce que la vie a raté. Elle laisse surgir l'événement, celui qui permet, non pas de changer le passé, mais d'en modifier les effets par le renouvellement du récit. Mon expérience et celle d'autres qui m'accompagnent dans mon exercice témoignent de changements psychiques profonds. Il me semble difficile de théoriser la question du changement. Je ne pourrais en indiquer ce qui en a été l'opérateur puisqu'il s'inscrit dans la singularité d'une histoire et d'une rencontre ${ }^{12}$. Mais pourtant, ces jeunes changent. Plus ils assument leur histoire dans les détails de ses aléas par la mise en récit, plus ils sont en mesure de s'affranchir de ces aléas. Ils peuvent s'accorder le droit d'advenir, pétris de leur passé, mais aussi ouverts à l'inconnu de ce qui n'a pas encore eu lieu tout en se tenant dans le lien à l'autre qui a résisté aux multiples attaques qui ont pu se jouer dans la prise en charge. Faire confiance à l'autre, ou plus exactement retrouver la confiance en l'autre et en soi, ne serait-ce pas une définition possible de l'autonomie? L'autonomie, ce n'est pas faire ce que l'on veut, comme on l'entend très souvent, encore moins faire tout seul comme cela est parfois avancé par les jeunes dans un relent de toute puissance imaginaire qui répond en écho à la détresse infantile réactualisée dans le désarroi adolescent. "Auto Nomos", les propres lois. L'autonomie, c'est obéir aux lois que l'on s'est données, lois qui font contenant et délimitent les zones frontalières par rapport aux autres. Ce n'est pas une visée, un but recherché, mais un effet. L'autonomie, maître mot de toute prise en charge, à tel point qu'il est nécessaire parfois de s'en méfier pour qu'il ne se vide pas de son sens, l'autonomie donc n'est pas étrangère à la psychanalyse telle que Serge Leclaire la donnait à entendre: «le but d'une analyse c'est de parler enfin à la première personne»! (Dufour, 2007)

Et pour que cette première personne parle enfin cela prend du temps ${ }^{13}$. Surtout si elle n'a pas été habituée à cet exercice là. Les jeunes qui sont pris en charge sont portés par différents discours. Celui de la famille, de l'ASE 
(qui se prend parfois pour la famille), de l'éducateur, etc. La liste n'est pas exhaustive. Ils les parlent comme on parle un idiome étranger. Il est nécessaire de les dégager de ces discours, parfois assourdissants, pour qu'advienne le leur. Là encore, parler est un risque!

Il y a quelques années, je suis allé au théâtre voir une adaptation Des enfants du Paradis. Quelle ne fut pas ma surprise, au lever de rideau, de voir tous les personnages déclamer leurs textes dans une saisissante cacophonie. Puis, un à un, ils se retirèrent côté cour et côté jardin pour réapparaître au moment opportun, laissant les deux personnages principaux commencer la narration. J'avais trouvé, dans cette mise en scène, une belle illustration du processus analytique.

\section{Le théâtre de Corentin}

Corentin rêve. "Je suis assis sur une chaise", me dit-il. "Dans une salle de réunion. La salle 21, je m'en souviens bien. Je préside l'assistance et je vous fais face." Dans tous les sens du terme me semble-t-il. "Je suis en colère. Je ne sais plus pourquoi mais je suis en colère contre vous. Je vous crie dessus et vous ne réagissez même pas. »Corentin est gêné de parler de ce rêve. Comme s'il n'avait pas le droit d'évoquer ce ressentiment à mon encontre ou du moins à ce que je viens représenter dans son rêve. Car s'il y a déplacement, il y a aussi condensation. Mais avant d'interpréter ce rêve, il me faut revenir sur ce qui le conditionne.

Corentin est pris en charge par l'ASE depuis l'âge de 7 ans. Suite au divorce de ses parents, toute la fratrie a été placée, excepté le frère aîné qui est resté avec la mère. Corentin a d'abord été placé en foyer, le temps pour sa mère d'arranger sa situation "financière». Elle devait revenir au mois de mars. Dix ans plus tard, il l'attend toujours. La marque de la première déconvenue est vive, acérée. Elle est toujours écrite au présent. Le temps s'est arrêté là, sur les marches $d u$ perron du foyer où il attendait chaque week-end le retour de sa mère. Les années se sont succédées. Du foyer d'urgence, Corentin a été pris en charge par une famille d'accueil. Puis la puberté venant, il a été orienté vers un nouveau foyer pour adolescents. Mais de l'adolescence, quid? Corentin est resté au seuil. Malgré ses 17 ans, il attend. Passivement. Il est ainsi orienté vers une association qui prend en charge des jeunes entre 16 et 21 ans.

Je l'ai accompagné durant quatre ans. Ses ${ }^{14}$ quatre années qui le transbahutaient inexorablement jusqu'à ses 21 ans. Le travail d'élaboration, d'énonciation sans laquelle il n'y a pas de subjectivation, lui a permis de se séparer de cette mère qui n'était pas venue le chercher. Progressivement, il a pris de la distance. Si la mesure de placement avait été une distanciation physique (parfois 
nécessaire), la cure a induit une autre distanciation, plus psychique. Il a pu faire taire les différents discours dont il était porteur pour les convoquer au moment opportun et interroger ses imagos jusqu'à en être parfois écœuré. Mais ne fautil pas en passer par là pour apprendre vraiment qui est l'autre? Il a pu réorganiser les modalités de relation aux autres afin d'y trouver un équilibre, son équilibre, dans le cadre précis de sa prise en charge. Et, enfin, il a pu reprendre des études, trop longtemps arrêtées par l'espoir et l'attente qui l'accompagne. Quatre années pour reprendre et poursuivre son adolescence là où elle s'était arrêtée. Quatre années pour quitter les marches de ce perron, érodées par le temps.

Nous savions tous deux que nous étions tenus dans le temps qui rythmait les séances au gré des renouvellements de contrat "jeune majeur", promettant une bouffée d'air thérapeutique ou le surgissement renouvelé de l'angoisse d'abandon. D'une certaine façon, Corentin allait «suffisamment mal» pour qu'on le laisse poursuivre son processus de séparation inscrit en filigrane dans le travail thérapeutique. Là encore, les jeunes ne sont pas dupes!

Mais à l'orée des 21 ans qui s'annonçaient avec l'arrivée du printemps, la colère montait et le dépassait. Qu'allait-il, de lui, advenir? De ce Je formulé de séance en séance, Corentin ne s'en sentait pas assuré. Le rêve prenait là tout son sens.

Si le 21 de la salle de réunion n'est pas sans le renvoyer au 21 de son âge qui annonce une nouvelle séparation, dont on sait qu'elle réactualise toutes celles qui n'ont pas été symbolisées, il peut permettre enfin qu'une parole s'affirme. Qu'il préside. Qu'il dise ce qu'il pense à cette assistance constituée de toutes ces personnes qui ont été plus ou moins là : sa mère, sa famille, les professionnels de l'ASE, moi, etc. Qu'il dise ce qu'il en pense de cette assistance qui n'est autre que l'ASE, anciennement Direction Des affaires sanitaires et sociales, anciennement Assistance publique. L'assistance, c'est aussi une certaine condition, que d'aucuns, dans leur logique moralisatrice, qualifient trop rapidement d'assistanat. Durant des années, Corentin avait pu bénéficier d'un dispositif qui lui avait permis de se reconstruire. Après 21 ans, ce dispositif s'étiolerait sans qu'un autre ne vienne le remplacer. Le confort, somme toute relatif, que Corentin avait pu trouver dans sa prise en charge allait disparaître pour que lui succèdent les doutes et les incertitudes liés aux conditions de vie des jeunes en France entre 21 et 25 ans ${ }^{15}$.

L'un des paradoxes de toute prise en charge tient là, dans ce qui leur est proposé et ce qui leur reste au sortir de celle-ci. Beaucoup de jeunes 
expriment une certaine colère à avoir été "protégés » de cet après. Corentin n'échappait pas à la règle. La colère était dans la séparation et dans ce qu'elle pouvait induire comme angoisse. Pendant les deux tiers de sa vie, il avait été pris en charge. Toutes ses démarches avaient été soutenues et/ou conduites par l'ASE et les différents professionnels qu'il avait rencontrés. Si 21 ans pouvait évoquer chez lui une certaine liberté, elle n'était pas sans être teintée d'angoisse. D'autant plus que je ne pouvais rien y faire, comme tant d'autres. D’autant plus que je ne réagissais même pas!

Corentin a un rêve. À 21 ans, il doit présider sa vie, marquée par son passage à l'ASE, et ce sans que plus personne ne vienne lui dire ce qu'il devrait faire. La colère s'est déplacée. Elle s'est actualisée au cours du travail thérapeutique et le ressentiment, tel qu'il l'avait gardé à l'encontre de sa mère mais qui était resté tu, il peut enfin l'exprimer sans que cela ne le détruise, sans que cela ne détruise l'autre. Le lien n'est pas rompu même s'il se distend.

Quelques années plus tard, Corentin a "refait sa vie». Il a rencontré une jeune femme avec laquelle il s'est installé. Il a trouvé du travail malgré les lazzis et les quolibets de son entourage quant au choix de métier, éducateur de rue en l'occurrence. Et il envisage de quitter "enfin» la région parisienne pour aller s'installer en province, loin de ce qu'il a toujours connu et qui l'a plus desservi qu'autre chose. Sa famille n'est pas d'accord avec ce projet. "Elle s'en arrangera...»dit-il.

Le défi pour ces jeunes est le suivant: lier les demandes qui leur sont adressées, notamment par l'ASE, et maintenir vivant le processus de subjectivation. Il tient en cela, ce défi, parce que les jeunes rencontrent un analyste et que cette rencontre les engage tous deux, l'un dans son symptôme et son désir - qui peuvent se confondre parfois, si ce n'est souvent-, l'autre dans son éthique et aussi son désir - qui ne doivent pas se confondre pour autant. Toute la gageure dans ce type d'accompagnement est de lier, autant que faire se peut, le temps social de la prise en charge scandée par les renouvellements (tous les 6 mois au mieux) et par les demandes, lorsqu'elles ne sont pas exigences, au temps psychique, ce temps de l'inconscient dont on sait depuis Freud qu'il ignore le temps.

Pour reprendre une question posée dans l'argumentaire, «en acceptant cette implication "hors les murs", à l'extérieur de son cabinet, le psychanalyste est confronté aux exigences simultanées d'une double temporalité, celle du psychisme et celle de la réalité externe [...] Le travail analytique s'en trouvera- 
t-il simplement modulé, ou complètement inaccessible?», question qui a orienté mon travail d'écriture, je soutiens que c'est justement parce qu'un jeune pris en charge rencontre un analyste que quelque chose se produit et que cette production, psychique, a des effets dans la réalité externe. Mais aussi, parce qu'un analyste prend en compte cette réalité externe, cela induit des modifications psychiques chez ce jeune. L'un ne va pas sans l'autre.

En psychanalyse le patient vient avec ses symptômes, le thérapeute avec sa technique, et, si les choses ne se passent pas trop mal, ils finiront tous deux par sortir de leur cachette ${ }^{16}$.

C'est là l'engagement de l'analyste notamment dans son rapport au symptôme; symptôme qui sous-tend et soutient une position subjective. C'est là que l'analyste interpelle le sujet, le jeune pris en charge, dans le savoir qu'il a de son histoire même s'il ne veut rien en savoir. Travailler avec ces jeunes comme analyste, lorsque bien sûr les conditions sont réunies pour remplir cette fonction, c'est leur permettre de rompre les amarres qui les entravent depuis longtemps, amarres soutenues par les différents discours desquels ils sont objet, au risque d'un désarroi dont il leur faudra faire la traversée pour s'en libérer. «Sortir de sa cachette» permet de renouer le temps social au temps psychique, de faire que Kairos surprenne Chronos et en modifie le cours pour que le jeune intègre son désir à la réalité extérieure sans forcément s'y adapter.

De ce fait, l'intervention d'un psychanalyste peut compliquer considérablement une prise en charge, du moins dans un premier temps. Elle peut compliquer plus que l'on ne le souhaitait, plus que l'on ne l'escomptait. Le «on » de rigueur est là bien indéfini. Il renvoie au jeune, à l'association mandatée, à l'ASE, si ce n'est aux trois simultanément. Ce n'est pas en soi une complication volontaire, attendue voire pensée, c'est un effet de la rencontre et de l'élaboration qui se développe dans le discours psychanalytique, qui n'est autre que celui du patient.

Je n'idéalise pas la psychanalyse ${ }^{17}$. J'en connais les écueils comme son efficace. D'autres thérapeutiques pourraient fonctionner tout autant. Néanmoins, c'est à partir du corpus freudien que se fonde ma pratique et c'est à partir de ce corpus et de la rencontre avec tel ou tel jeune qu'elle se modifie. Dans ce type de prise en charge, ce qui peut moduler la psychanalyse, sans la rendre inaccessible pour autant, c'est la dimension du temps, d'un temps fixé par la prise en charge et par la loi qui la conditionne. 


\section{Pour (ne pas) conclure}

Un peu de polémique, voire de politique. Lorsque je reçois un jeune pris en charge par l'ASE, j'ai toujours en tête ce point d'arrêt inexorable que seront les 21 ans. Je sais, même si cela peut s'arrêter avant ou si certains jeunes peuvent poursuivre leur travail par la suite, que la rencontre est marquée par cette date. C'est la ligne d'horizon. C'est parfois ma ligne d'horizon, ne seraitce que pour maintenir ma capacité à penser. Et j'en fais part aux jeunes que je reçois. Je mentionne ce temps et les scansions qui le composent pour ne plus avoir à trop y penser ensuite. Enfin, presque... Le temps social d'une prise en charge n'a en soi rien à voir avec le temps psychique de la subjectivation. Néanmoins, le premier vient encadrer le second. C'est pourquoi il nous faut nous hâter avec lenteur, prendre la mesure des deux temps et les (faire) respecter, parce que si ce ne fut pas au détriment du second dans la situation de Corentin, ça l'a été pour d'autres.

La disparition du symptôme, l'insertion sociale ou professionnelle, «l'autonomie », sont intimement liées au travail d'élaboration psychique, à ce nouveau positionnement d'un sujet dans son histoire. Les prendre comme ils se présentent, sans les mesurer avec ce qui s'est déployé sur la scène psychique, c'est rater probablement différents enjeux, c'est prendre l'arbre pour la forêt ou encore des «vessies pour des lanternes ${ }^{18}$ ». Les jeunes ne sont pas sans savoir que l'obtention d'un emploi peut mettre un terme à leur prise en charge. Tous le savent. Si certains le souhaitent, d'autres le redoutent parce qu'ils ne sont pas prêts psychiquement à cet arrêt. Si la fin d'une prise en charge est toujours un moment délicat, où peuvent se répéter les raisons qui ont fondé cette prise en charge, elle se lie aussi avec la problématique de la résolution de l'adolescence. Cette dernière pose question. D'aucuns (rares) l'associent à la majorité. D'autres la poussent jusqu'aux environs de 25 ans. Certains l'imaginent jusqu'à l'âge où leurs propres enfants auront atteint à leur tour l'adolescence...

L'avènement de la puberté inaugure des transformations qui doivent amener la vie sexuelle infantile à sa forme normale définitive. (Freud, 1905/1987, 143)

L'expérience clinique, tant avec ces jeunes pris en charge qu'avec d'autres jeunes «adultes», me fait penser que cette forme sexuelle normale et définitive, à laquelle se réfere Freud, se situe habituellement vers l'âge de 21 ans, un âge où l'on est souvent capable d'établir des relations, amoureuses ou non, 
stables. Je ne me plierai pas ici à une distinction concernant la différence des sexes, passionnante s'il en est, mais hors de propos. 21 ans, donc! Vous me direz que c'est justement l'âge auquel s'arrête une prise en charge. Nul besoin de polémiquer... Effectivement si la prise en charge se poursuit jusqu'à cet âge, cela peut permettre que suffisamment d'éléments se mettent en place sur la scène psychique pour qu'un jeune poursuive sa vie sans trop de difficultés, ou du moins en ayant pu en régler quelques-unes. Mais, parce qu'il y a souvent un mais, trop souvent, les prises en charge qui perdurent jusqu'à 21 ans se font de plus en plus rares et doivent la plupart du temps répondre à des normes drastiques d'insertion sociale et professionnelle. L'étymologie du mot placement renvoie au monde de la finance, et d'une manière ou d'une autre, cela fait retour.

Nous oublions que nous demandons à des jeunes au bord de la désocialisation, pour lesquels le recours à l'acte est parfois un impératif, qui sont marqués par des carences affectives majeures, de faire plus dans un laps de temps précis que nous en demanderions à d'autres jeunes vivant chez leurs parents (et) considérés, à tort ou à raison, comme plus stables. N’y a t-il pas là un vrai paradoxe sur lequel il serait avisé de réfléchir?

Du Lièvre et la tortue de Jean de La Fontaine, on sait dès le premier vers que « rien ne sert de courir; il faut partir à point ». Des Jeunes et de l'ASE, on pourrait donc dire que : «rien ne sert de courir, il faut arriver à temps».

\author{
Nicolas Peraldi \\ 8 , rue de Belzunce \\ 75010 Paris \\ nicolas. peraldi2@free.fr
}

\title{
Notes
}

1. Cet article est une version remaniée et développée d'une conférence, «Parce que je me retourne vers le passé, je vois l'avenir ", tenue lors du colloque organisé par l'Association Notr'Asso sur L'idée psychanalytique dans l'insertion des jeunes en rupture, Paris, le 14 octobre 2010.

2. Par commodité de langage, j'utiliserai l'acronyme ASE.

3. Pour reprendre le titre du livre d'Aichhorn, Jeunesse à l'abandon où Freud, dans sa préface, évoque pour la première fois l'impossible des trois métiers que sont éduquer, gouverner et psychanalyser.

4. Je prends quelques libertés avec la grammaire puisque le verbe procrastiner n'existe pas.

5. Certains le sont avant même de naître, leurs parents biologiques n'envisageant pas de les garder.

6. La définition des missions de l'ASE a été reformulée par le décret n ${ }^{\circ} 75-96$ du 18 février 1975 puis a été complétée par le loi du 5 mars 2007 réformant la Protection de l'enfance. 
Il y est dit, en substance, que jusqu'à l'âge de 21 ans, toute personne majeure (ou mineure anticipée) peut solliciter, soit une prise en charge par l'ASE, soit une action de protection judiciaire auprès du Juge pour enfants. Lorsqu'il est recouru aux mesures d'aide sociale aux jeunes majeurs, le projet individualisé est concrétisé par un contrat écrit et signé par le jeune et au service de l'ASE. Ce contrat, d'une durée d'un an maximum, ce qui est une denrée rare, est renouvelable. Il doit faire référence à des objectifs précis à atteindre et à des moyens concrets de mise en œuvre. L'insertion professionnelle et l'accès à l'autonomie doivent rester une priorité. La prise en charge de l'ASE prend fin soit à l'expiration d'un délai fixé en accord avec l'intéressé, soit lorsque celui-ci a atteint l'âge de 21 ans, soit à tout moment à l'initiative du Conseil général ou de plein droit du bénéficiaire.

7. Depuis 1793 et jusqu'au 5 juillet 1974, la majorité était fixée à 21 ans. Logiquement, l'abaissement de la majorité à 18 ans aurait dû conduire l'ASE à stopper son intervention à cet âge. Mais, sous la pression des professionnels du secteur, le législateur a convenu qu'il pouvait être nécessaire de poursuivre jusqu'à 21 ans, comme précédemment. D'où ce statut hybride de « jeune majeur» et le fait qu'entre 18 et 21 ans, l'ASE peut intervenir sans être pour autant obligée de le faire.

8. Lors du colloque susmentionné, Didier Lauru avait eu son bon mot de «neutralité engagée» nécessaire avec les adolescents. Cet oxymore est d'autant plus nécessaire avec les jeunes de l'ASE me semble-t-il.

9. Je joue ici d'une expression française, «à cor et à cri », puisque le désir, on le sait, peut s'exprimer dans le corps lorsqu'il se fait maladie, par le corps lorsqu'il devient agir...

10. La loi du 2 janvier 2002 qui gère les établissements sociaux et médico-sociaux a introduit cette nouvelle catégorie sous forme de paradigme qu'est l'usager. S'il est au centre des préoccupations, il est devenu le socle sur lequel doit se fonder toute pratique. La Langue Médico Sociale (LMS), pour reprendre la notion de Yann Diener, a fait son office.

11. À ce propos, on peut se référer à un précédent article: «Mauvais Fils» (Peraldi, 2011).

12. J'ai essayé dans l'article sus mentionné de relater les enjeux et ce qui a pu opérer dans l'accompagnement d'un jeune majeur pris en charge par l'ASE.

13. M. Cooperman disait qu' « une psychanalyse, ça prend deux semaines, mais il faut plus de deux ans pour arriver à ces deux semaines » (Davoine F., Gaudillière, J.M., 2006, 346).

14. Ceci n'est pas une faute d'orthographe, mais une figure de style comme une insistance (lexicale) sur le fait qu'il s'agissait du temps vécu par Corentin.

15. À ce jour, il n'existe en France aucune aide possible pour les jeunes entre 21 et 25 ans. Soit ils ont réussi à mettre en place une certaine insertion sociale et professionnelle avant leurs 21 ans, soit ils devront se débrouiller comme ils peuvent jusqu'à leurs 25 ans. Une récente enquête télévisée, sur une grande chaine de télévision française réputée pour ses scoops plus ou moins avérés, voudrait montrer que $50 \%$ des jeunes passés par ce que l'on appelle aujourd'hui l'ASE finissent SDF. D'un acronyme à un autre. Si je reste dubitatif sur les statistiques, je ne peux qu'être conscient, ayant travaillé avec des personnes dites SDF, du nombre important chez ces personnes d'anciens de la DDASS. Ce creux, lorsqu'il n'est pas abyme, entre 21 et 25 n'y ait peut-être pas pour rien... On peut dès lors s'inquiéter lorsque l'on sait que le nombre de contrat jeune majeur se réduit comme peau de chagrin! Quelle marge de manœuvre auront ces jeunes, livrés à eux-mêmes, vivant d'expédients divers, oscillant entre le sujet en exclusion jonché sur le trottoir et celui en privation qui fraye avec la délinquance dans l'attente désespérée des 25 ans qui leur permettront de bénéficier d'un Revenu de solidarité active? Si les contrats jeune majeur ont un coût, indéniable, n'est-il pas en fin de compte plus onéreux, à la longue pour la société, de laisser dériver certains jeunes qui auraient pu bénéficier d'un soutien plus qu'économique jusqu'à leur 21 ans (au moins) ? Des questions politiques s'il en est, au vrai sens du terme, 
avec lesquelles le clinicien que je suis doit travailler sans militantisme mais sans indifférence, tant le temps est présent dans ce type de prise en charge.

16. Encore un aphorisme de M. Cooperman, cité par D. Gaucher, lors d'une intervention («La psychanalyse guérit-elle?») à l'occasion des rencontres organisées par la revue Pratiques, le Centre du Vivant (Paris VII) et l'Association Diderot.

17. Encore faudrait-il interroger le singulier de la formulation. La psychanalyse existe-t-elle? À y croire, on instaure du dogme et des maîtres. On clôt la créativité, l'inventivité qui opère dans chaque cure. On ne se laisse pas surprendre comme Freud le préconisait à Ferenczi. On applique un mode d'emploi, transmis de génération en génération d'analystes. Loin de servir la psychanalyse, très loin de servir les patients qui ne servent eux dès lors qu'à confirmer la théorie qui préside à leur « cure», cela conforte aussi ses détracteurs. N'y a t-il pas en fait autant d'analyses qu'il y a d'analystes, voire autant d'analyses qu'il y a d'analysants... Sur ces questions, on peut lire l'ouvrage passionnant d'Anne Millet: Psychanalystes, qu'avons nous fait de la psychanalyse?

18. Expression populaire qui signifie: «se faire des illusions grossières sur des choses ou des gens, se tromper lourdement dans ses appréciations ».

\section{Références}

AICHHORN, A., 1925, Jeunesse à l'abandon. Psychanalyse et éducation spécialisée, Nîmes, Champs Social, 2005.

DAVOINE, F., GAUDILLIÈRE, J. M., 2006, Histoire et trauma, la folie des guerres, Paris, Stock.

DIENER, Y., 2011, On agite un enfant. L'État, les psychothérapeutes et les psychotropes, Paris, La Fabrique.

DOLTO, F., 1972, Au jeu du désir, les dés sont pipés et les cartes truquées, in Au jeu du désir, Paris, Seuil, 1981.

DUFOUR, D. R., 2007, Le divin marché, la révolution culturelle libérale, Mayenne, Denoël.

FREUD, S., 1900, Le réveil par le rêve, in L'interprétation du rêve, Euvres complètes Psychanalyse, Tome IV, Paris, PUF, 2003.

FREUD, S., 1905, Les transformations à la puberté, in Les trois essais sur la théorie sexuelle, Paris, Gallimard, 1987.

FREUD, S., 1932, La décomposition de la personnalité psychique, $\mathrm{XXX}^{\mathrm{e}}$ conférence, in Nouvelles conférences d'introduction à la psychanalyse, Paris, Gallimard, 1984.

FREUD, S., 1937, Analyse avec fin, analyse sans fin, in Résultat, Idées, Problèmes, Tome 2, Paris, PUF, 1985.

GAUCHET, D., 2006, La psychanalyse guérit-elle? http://www.smg-pratiques.info/IMG/pdf/ Gaucher_la_psychanalyse_guerit-elle_7_novembre_2006.pdf

MILLET, A., 2010, Psychanalystes, qu'avons-nous fait de la psychanalyse?, Paris, Seuil.

PERALDI, N., 2011, Mauvais fils, Adolescence, 29 (4), 765-778.

REIK, T., 1935, Le psychologue surpris, deviner et comprendre les processus inconscients, Paris, Denoël, 1976.

ZYGOURIS, R., 2006, Le lieu de la plus grande force, Pratiques, les cahiers de la médecine utopique, $\mathrm{n}^{\circ} 33,16-22$. 